\title{
PET/CT Imaging in Mouse Models of Myocardial Ischemia
}

\author{
Sara Gargiulo, ${ }^{1,2}$ Adelaide Greco, ${ }^{1,2}$ Matteo Gramanzini,, 2 \\ Maria Piera Petretta, ${ }^{1,3}$ Adele Ferro, ${ }^{1}$ Michele Larobina, ${ }^{1}$ \\ Mariarosaria Panico, ${ }^{1}$ Arturo Brunetti, ${ }^{1,2}$ and Alberto Cuocolo ${ }^{1,2}$ \\ ${ }^{1}$ Department of Biomorphological and Functional Sciences and Institute of Biostructures and Bioimages \\ of National Council of Research, University of Naples Federico II, Naples, Via S. Pansini 5, 80131 Naples, Italy \\ ${ }^{2}$ CEINGE-Biotecnologie Avanzate scarl, Via G. Salvatore 486, 80145 Naples, Italy \\ ${ }^{3}$ Department of Internal Medicine, Cardiovascular and Immunological Sciences, University of Naples Federico II, \\ Via S. Pansini 5, 80131 Naples, Italy
}

Correspondence should be addressed to Sara Gargiulo, sara.gargiulo@ibb.cnr.it and Alberto Cuocolo, cuocolo@unina.it

Received 4 November 2011; Revised 16 December 2011; Accepted 30 December 2011

Academic Editor: Andrea Vecchione

Copyright (C) 2012 Sara Gargiulo et al. This is an open access article distributed under the Creative Commons Attribution License, which permits unrestricted use, distribution, and reproduction in any medium, provided the original work is properly cited.

\begin{abstract}
Different species have been used to reproduce myocardial infarction models but in the last years mice became the animals of choice for the analysis of several diseases, due to their short life cycle and the possibility of genetic manipulation. Many techniques are currently used for cardiovascular imaging in mice, including X-ray computed tomography (CT), high-resolution ultrasound, magnetic resonance imaging, and nuclear medicine procedures. Cardiac positron emission tomography (PET) allows to examine noninvasively, on a molecular level and with high sensitivity, regional changes in myocardial perfusion, metabolism, apoptosis, inflammation, and gene expression or to measure changes in anatomical and functional parameters in heart diseases. Currently hybrid PET/CT scanners for small laboratory animals are available, where CT adds high-resolution anatomical information. This paper reviews mouse models of myocardial infarction and discusses the applications of dedicated PET/CT systems technology, including animal preparation, anesthesia, radiotracers, and images postprocessing.
\end{abstract}

\section{Introduction}

Cardiovascular diseases, such as coronary heart disease (CHD), are common causes of morbidity and mortality in developed countries. Currently CHD is a relevant cause of permanent disability in workers and congestive heart failure, which is frequently secondary to myocardial infarction (MI). Despite progress in the diagnosis and treatment of CHD, appropriate animal models play a central role to investigate the mechanisms involved in the pathogenesis and for the advancement of diagnosis and therapies. Rodents, in particular mice, are often used in cardiovascular research, since they are not expensive, easy to handle and to house, are easily prone to genetic manipulations and show anatomic similarity in developmental and postnatal heart compared with humans. Therefore, conventional wild-type and genetically altered mice are becoming interesting models for better understanding the pathogenesis and improvement in diagnosis, prevention, and therapy of CHD in humans. Noninvasive in vivo imaging has acquired a critical role in mouse cardiovascular research; however, the small size and rapid rate of the mouse heart pose significant challenges for cardiac imaging, requiring expensive equipment and established expertise (Figure 1). Since the left ventricle wall is thinner than $1 \mathrm{~mm}$, high spatial resolution is mandatory. At the same time, the rapid heart movement requires high temporal resolution. Table 1 indicates several imaging techniques that are currently used for morphological and functional phenotyping of mouse cardiovascular system, including high-resolution ultrasound, $\mathrm{X}$-ray computed tomography (CT), magnetic resonance, and nuclear medicine procedures. Molecular imaging with positron emission tomography (PET) allows evaluating, in a non-invasive and longitudinal manner, biological processes 
TABLE 1: Principal characteristics of small animal imaging techniques in cardiovascular research.

\begin{tabular}{|c|c|c|c|c|}
\hline $\begin{array}{l}\text { Imaging } \\
\text { technique }\end{array}$ & $\begin{array}{l}\text { Physical } \\
\text { principle }\end{array}$ & $\begin{array}{l}\text { Spatial } \\
\text { resolution }\end{array}$ & Advantages & Disadvantages \\
\hline UBM & $\begin{array}{l}\text { High frequency } \\
\text { sound waves }\end{array}$ & $50 \mu \mathrm{m}$ & $\begin{array}{l}\text { Real-time cardiovascular } \\
\text { morphofunctional assessment }\end{array}$ & $\begin{array}{l}\text { Strongly operator dependent, } \\
\text { difficult assessing of right } \\
\text { ventricle }\end{array}$ \\
\hline CT & $\mathrm{X}$-rays & $100 \mu \mathrm{m}$ & Left ventricle morphology & Radiation dose \\
\hline MRI & $\begin{array}{l}\text { High intensity } \\
\text { magnetic field }\end{array}$ & $200 \mu \mathrm{m}$ & $\begin{array}{l}\text { High tissue contrast and } \\
\text { functional parameters }\end{array}$ & Most expensive and availability \\
\hline SPECT & $\begin{array}{l}\text { Gamma } \\
\text { emitters }\end{array}$ & $\begin{array}{l}\text { Max } 0.62 \mathrm{~mm} \\
\text { FWHM }\end{array}$ & $\begin{array}{l}\text { Molecular imaging, myocardial } \\
\text { metabolism and perfusion, } \\
10^{-10}-10^{-11} \mathrm{M} \text { sensitivity }\end{array}$ & $\begin{array}{l}\text { Low spatial resolution, radiation } \\
\text { dose }\end{array}$ \\
\hline PET & $\begin{array}{l}\text { Positron } \\
\text { emitters }\end{array}$ & $\begin{array}{l}\text { Max } 1 \mathrm{~mm} \\
\text { FWHM }\end{array}$ & $\begin{array}{l}\text { Molecular imaging, myocardial } \\
\text { metabolism and perfusion, } \\
10^{-11}-10^{-12} \mathrm{M} \text { sensitivity }\end{array}$ & $\begin{array}{l}\text { Low spatial resolution, radiation } \\
\text { dose }\end{array}$ \\
\hline
\end{tabular}

UBM: ultrasound biomicroscopy; CT: computed tomography; MRI: magnetic resonance imaging; SPECT: single-photon emission computed tomography; PET: positron emission tomography; FWHM: full width at half maximum.

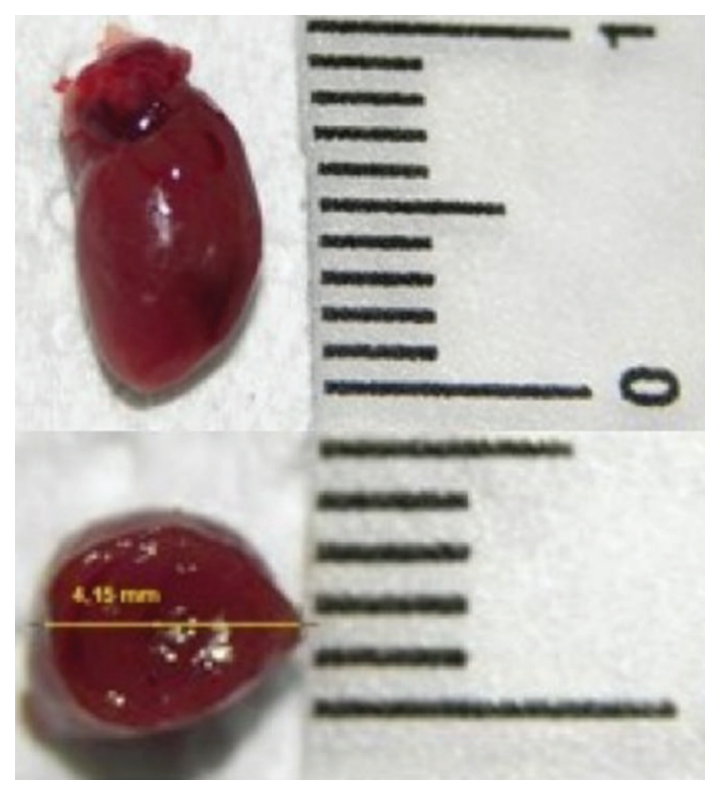

FIGURE 1: Longitudinal and transversal dimensions ( $\mathrm{mm}$ ) of mouse heart.

at a cellular and subcellular level in early steps of heart diseases, to perform quantitative analysis, to study apoptosis, angiogenesis, hypoxia, inflammation, receptor density, and gene expression. In spite of the great specificity and sensitivity of PET, molecular imaging approach can take advantage from simultaneous acquisition of morphological information for localization and quantification. Therefore, hybrid imaging with PET/CT systems is becoming the most used approach in cardiovascular imaging. In this paper we review mouse models of myocardial infarction and discuss the applications of dedicated PET/CT systems technology, including animal preparation, anesthesia, radiotracers, and images postprocessing.

\section{Models of Myocardial Infarction}

In an animal model the induced disorder should closely resemble the disorder in human. None of the currently available animal models can entirely reproduce the full spectrum of cardiovascular diseases in humans. Rodents, especially rats and mice, are commonly used in cardiovascular research because they are less expensive and are easier to handle and to house compared to large animals [1]. Since a broad variety of genetically modified mice are produced and knockin and knockout techniques are well developed on mice, mouse models are increasingly adopted to study cardiovascular human diseases and to identify new therapeutic targets. The most frequently used include MI or ischemia-reperfusion by ligation of the left coronary artery [2, 3]. Despite several anatomic differences, the value and clinical translatability of mouse models of cardiac diseases have been proven. The mouse heart is similar to the human concerning ventricular structure and valves. The major anatomical differences are seen in the atria and venous pole of the heart (Figure 2). In human, left atrium receives four pulmonary veins, while in the mouse the pulmonary veins join in a single vessel. During organogenesis, in human left cranial caval vein regresses and the proximal portion becomes the coronary sinus, while the distal portion gives rise to the ligament of Marshall and the oblique vein [4]. In the mouse, the left cranial caval vein does not regress and persist in postnatal life. These differences in venous tributaries into atrial chambers are the likely reasons for the relatively small size of atria in murine heart [5]. The mouse coronary anatomy has some differences compared to human. Mice have a single septal coronary artery, arising from separate ostia from the right sinus of Valsalva or as a proximal branch of the right coronary artery, while in man the interventricular septum is perfused by septal perforator arteries arising from the left anterior descending or posterior descending coronary arteries. Mouse left coronary artery does not divide proximally into left anterior descending and circumflex artery, but courses obliquely across the left ventricular (LV) free wall and branches in a variable 


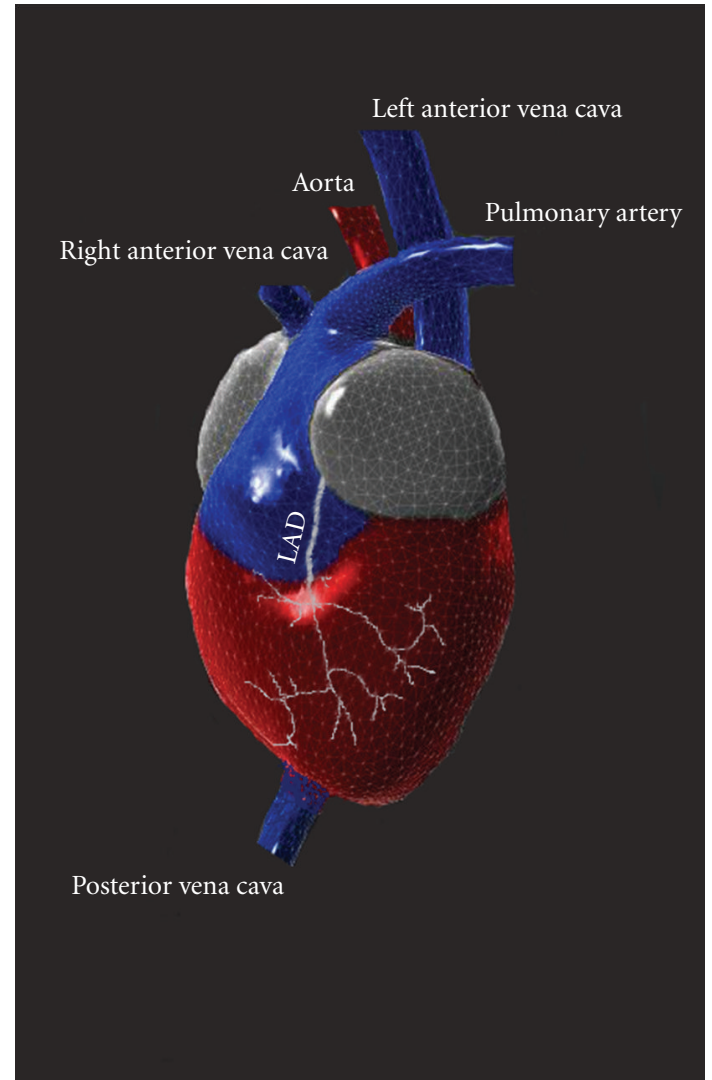

Figure 2: Tridimensional reconstruction of mouse heart, representing the peculiar features of venous pole and left coronary artery anatomy.

fashion, similar to the ramous intermedius artery in man. The right coronary artery branches proximal into a right ventricular and circumflex vessel supplying the LV posterior wall. Therefore, the distinct mouse coronary artery anatomy results in different regionality of infarction compared with human, because ligation of the left coronary artery in mice produces ischemia in the anterolateral, posterior, and apical regions of the heart, sparing the septum [2]. Also the anatomy of conduction system is different from human, because the sinoatrial node is located in the superior caval vein above its junction with the right atrium, not in the atrium itself [6]. Mouse model of myocardial ischemia has highlighted the mechanisms of infarct and LV remodeling and is often the first choice for testing new therapies such as stem cell myocardial repair. Animal models mimicking the characteristics and development of human MI and heart failure related to $\mathrm{CHD}$ have been developed with various strategies and methods. In humans CHD is related to chronic narrowing by atherosclerotic plaques or by acute occlusion by thrombosis of coronary arteries. In animals, atherosclerosis can be reproduced inducing hypercholesterolemia by fat/cholesterol- rich diet or by genetic manipulation. However, time point and site of coronary occlusion occur accidentally in these models and, in the latter case, the etiology is different from that naturally occurring in humans. Various pathologic conditions mimicking human cardiovascular diseases can be induced by microsurgery. For example, surgical induction of narrowing or occlusion of coronary arteries allows facilitating a precise timing, location, reproducibility, and extent of coronary disease. According to the duration and extent of coronary blood flow impairment, surgical models can be classified as MI, cardiac ischemia-reperfusion injury, and chronic cardiac ischemia. In recent years, the mouse is the species increasingly used to characterize MI induced by coronary artery ligation. This is the major animal model of LV dysfunction progressing to failure. A main technical challenge to perform cardiovascular surgery in mice is the little size of mouse heart and coronary arteries. A microscope is required to clearly detect and bind the small left coronary artery. Moreover, dedicated microsurgical instruments, thin sutures and needles, and a customized mouse ventilator should be employed. To perform surgery, the mouse is anesthetized with intraperitoneal or inhalant agents. It is important to not disturb the animal prematurely, since this will induce stress and affect the quality of the subsequent procedure. After an adequate depth of anesthesia is attained, the mouse is fixed in a supine position on a heating table to prevent hypothermia and is intubated to provide assisted ventilation. Tracheal intubation of mice is challenging, due to their small mouth and then the difficulty to visualize vocal cord opening. If performed by nontrained operators, there is a high risk of pharyngeal or tracheal damage, such as perforation or stenosis. Intubation can be made with a customized tracheal cannula or a modified 18-20 gauge intravenous Teflon catheter, of about $25 \mathrm{~mm}$ of length, inserted through the oral cavity or after tracheotomy/tracheostomy. Visualization of vocal cord opening can be facilitated with appropriate positioning and using dedicated "self-made" device [7-12]. A drop of $1 \%$ lidocaine can be applied on the tip of the tracheal cannula to reduce laryngeal reflex. After intubation, the mouse is connected to a mouse ventilator, providing oxygen 2 liters/min and in case an inhalant anesthetic, with a tidal volume of $200 \mu \mathrm{L}$ and a respiratory rate of about 133 breaths/min. Prior to the incision, the chest is trichotomized and disinfected with iodopovidone solution, and $0.1 \mathrm{~mL}$ of $0.1 \%$ lidocaine is injected subcutaneously. The thoracotomy is performed by a transverse $5 \mathrm{~mm}$ incision of the left fourth intercostal space, $2 \mathrm{~mm}$ away from the left sternal border. It is important to avoid bleeding caused by damage intercostal or internal thoracic veins. Chest retractor is gently inserted to facilitate heart exposition. The pericardial sac is gently opened, taking care to avoid the rupture of the wall of the left superior vena cava. The left coronary artery is visualized as a pulsating bright red vessel, running in the middle of the heart wall from the left atrium toward the apex. Ligation is performed with a 7-0 suture passed with a thin needle under the artery, 1-2 mm lower than the tip of the left auricle, which induces $40-50 \%$ ischemia of the left ventricle. It is important to not enter the cavity of the ventricle with the needle, but also to not be too superficial, as suture will cut through the wall ventricle. Blanching of the tissue distal to the ligation can recognize the occlusion of the artery. It is important to overinflate lungs by shutting off with a finger the outflow of the ventilator for 1-2 seconds. In fact, the 
lungs could be partially compressed by the retractor and their collapse could result in respiratory distress and poor recovery after surgery. The chest cavity is closed by bringing together the 4th and the 5th ribs, muscles, and skin with 70 absorbable sutures. The duration of the whole procedure is reported of about 20 minutes for trained personnel. The permanent occlusion of left coronary artery is followed by an extensive myocardial remodeling, a hypertrophy of the viable myocardium and thinning of the necrotic area [13]. Genetic background, age, and sex of animals should be considered because they can influence both infarct size and the disease progression $[14,15]$. Similar to the MI animal models, to perform ischemia-reperfusion models the left coronary artery is legated, but a small plastic tube is placed between the legated vessel and the node, allowing for easier and safe relief of the occlusion. Ischemia can be verified by sudden regional paleness of the myocardium, whereas reperfusion is verified by the appearance of hyperemia in the previously pale region. During the period of occlusion, the chest opening should be covered with a sterile gauze to prevent drying and loss of heat. This model is characterized by a high infiltration of inflammatory cells, attenuated fibrotic remodeling and angiogenesis in the necrotic area [13]. To minimize the influence of surgery on inflammatory process, a "closed chest" ligation model of myocardial ischemia-reperfusion injury in the mouse has also been developed: briefly, after thoracotomy, a thin suture is passed under the left coronary artery and the end of the suture are inserted into a piece of polyethylene-10 tubing and exteriorized through the chest wall [16]. Then thoracic incision is closed and ischemiareperfusion experiment is performed a few days later, pulling or releasing the suture ends, when inflammatory process induced by surgery is ended. The surgical murine model of MI is different from human pathological condition for several aspects: left coronary artery ligation produces in mice a transmural MI, whereas in human the development of collateral circulation and the potential cardioprotective effects of repeated mild ischemic events associated with CHD produce smaller, nontransmural infarct. In 1994, Miller and colleagues showed that "preconditioning" in the myocardium can induce a reduction of the infarct size of about $50 \%$. For preconditioning, the mouse undergoes three cycles of 5 minutes of artery occlusion, followed by 5 minutes of reperfusion, and then 30 minutes of artery occlusion followed by 2 hours of reperfusion. Mortality associated with MI induction in mice is about $10 \%$, due to acute malignant arrhythmias such as ventricular fibrillation after reperfusion or within 1 hour after surgery, that can be prevented by intraperitoneal injection of lidocaine $(6 \mathrm{mg} / \mathrm{kg})$ [13].

\section{Cardiac PET/CT Imaging}

Traditional cardiac imaging is based on detection of changes in the anatomy and physiological features of heart, such as blood flow or contractile function. Instead, molecular imaging is characterized by visualization and measurement of biochemical and cellular mechanisms of diseases, including altered energy metabolism, inflammation, apoptosis, thrombosis, and angiogenesis, thus improving early diagnosis and therapeutic approaches. Cardiac imaging in mice is difficult, due to the small ventricle volume and high heart rate. The use of molecular imaging in preclinical cardiovascular research has become possible by advances in imaging technology. In the last years, small animal PET systems with high spatial resolution and great sensitivity have been developed. The advantages of PET include higher sensitivity enabling dynamic imaging, creation of radiolabeled probes with natural radioisotopes that do not alter chemical behavior, well-established methods for attenuation correction, and the potential of absolute and semiquantitative accurate image quantification. PET allows measurement of functional processes like myocardial blood flow, cardiac output, metabolism of glucose or free fatty acid, or oxygen consumption in absolute units or, more simply, in a semiquantitative manner. Dynamic scans are required to measure the arterial input function and the tissue time activity curve. In humans and in larger animal models, these parameters are evaluated through repeated blood samples, or noninvasively by assessing the activity of the arterial blood pool in the LV cavity on PET images. Catheterbased arterial blood sampling is the gold standard method for measuring the input function in mice. Nevertheless, in mice arterial sampling is technically difficult because of the relatively small diameters and fragility of the mouse blood arteries [17]. In addition, the total blood volume of a mouse is very limited $(1.7 \mathrm{~mL})$, making repeated blood sampling impossible without affecting the homeostasis of the mice. To minimize blood loss and difficulty in sampling blood from a mouse during a dynamic small animal PET scan, a microblood sampling technique has been developed [18], but the number of blood samples that can be withdrawn is limited. To avoid these problems, various methods have been proposed to estimate the input function noninvasively, such as the derivation of liver time-activity curve from smallanimal PET dynamic images $[17,19]$ or measurement of blood pool activity from a LV volume of interest [20-24]. However, in small animal imaging, hearts and arteries are small compared with the scanner spatial resolution and in association with cardiac and respiratory motion causes blurring and partial volume effect artifacts. The correction of the partial volume related underestimation of the blood tracer concentration could be made determining recovery coefficient by different sizes rod phantoms [22] or using a four-dimensional virtual mouse phantom [25]. Moreover, the fast heart rate needs the correction for tracer recirculation. Since blood samples could not be obtained rapidly during the initial bolus transits through the central circulation, an early time point 20-35 seconds after injection has been proposed for blood-sampling-derived values. At this time, equivalent to several circulation times, the radiotracer concentration is relatively constant [20]. Implementation of clustering [26] or factor analysis algorithms [27] enables minimally invasive extraction of accurate input functions and myocardial time-activity curves from dynamic microPET images of rodents without the need to draw regions of interest, and overcoming the limitation related to partialvolume effect, spillover from the myocardium and motion 
blurring. Therefore, although the quantification of the metabolic rate of energetic substrates utilization improves the accuracy of measuring the extent of the infarct, in mice are commonly adopted semiquantitative approaches for analysis of the distribution of radiotracers. Measurement of infarct size is an important clinical goal for prognostic assessment and evaluation of therapeutic interventions [28]. Although the quantification of infarct size has been wellestablished in humans, this approach is more challenging in small animals due to their smaller size. In cardiovascular research, histological measurements are widely accepted as the gold standard for quantifying infarct size, also to validate imaging-based approach. Nevertheless, histomorphometry cannot provide serial measurements in the same animal over time, which are useful to monitor the evolution of a disease or the response to a treatment. Therefore, there is a great interest to develop an accurate and noninvasive method to evaluate infarct size in living mice, improving the knowledge of metabolic and functional changes related to MI and the efficacy of interventional, pharmacological, or molecular therapies. Several imaging-based approaches to measure infarct size have been developed on animal models. It has been reported that infarct size quantification in mice can be obtained in an accurate and repeatable way with CT [29], magnetic resonance [30], single-photon emission computed tomography (SPECT) [31], or PET [32]. In particular, PET has several advantages, such as high spatial resolution and sensitivity. Since tridimensional images can be reconstructed from PET datasets, this technique is more suitable than others, such as 2D echocardiography, to study MI models, which are characterized by greatly modified ventricular shape and irregular wall thickness [14]. Furthermore, with the advent of dedicated image reconstruction and quantification software, it is now possible to perform quantitative clinical measures in rodent heart.

Disadvantages of PET include the use of ionizing radiation and the physic intrinsic limit to the maximum spatial resolution achievable. Currently most micro-PET imaging systems are fused with micro-CT, to create hybrid-imaging systems that overcome the inherent low resolution of the very sensitive nuclear imaging techniques and provide the site of molecular tracers uptake in greater anatomic detail. Hybrid imaging systems will facilitate the translation of molecular imaging-based approaches to man. CT is also useful to perform accurate correction for partial volume errors. Disadvantages of CT include the use of ionizing radiation and the limited spatial resolution achievable in vivo, related to the radiation dose administered to the animals.

\section{PET Tracers for Models of Myocardial Infarction}

Alterations in myocardial substrate metabolism are critical in the pathogenesis of many cardiovascular diseases. MI is associated with numerous biochemical and functional changes in the necrotic tissue, in the area at risk, and in the remote myocardium. PET imaging can provide in vivo a noninvasive, serial and quantitative assessment of myocardial perfusion, metabolism, apoptosis, and gene expression. Moreover, electrocardiographic and respiratory gating PET imaging can also be used to analyze anatomic and functional parameters, such as LV shape, end-diastolic and end-systolic volumes, stroke volume, cardiac output, ejection fraction, and regional contractility [32]. The principal characteristics of the most commonly used PET tracers are illustrated in Table 2. PET radiotracers used for evaluation of myocardial blood flow include N-13 ammonia, rubidium-82, and O15 water [33]. However, their short half-life limits their widespread clinical use, because of the need for nearby cyclotron or generator. Among these, N-13 ammonia is the most widely used in preclinical studies, due to its relatively longer half-life of ten minutes, the high myocardium extraction, and reduced persistence in blood pool [34]. F18 fluorodeoxyglucose (FDG) is the most widely used PET tracer and its uptake provides an established method in clinical practice to measure tissue viability in patients with advanced CHD and impaired LV function. Compared with the other tracers, FDG is more readily available in most PET centers and allows for better resolution images [35]. FDG traces myocyte glucose uptake and phosphorylation and can be used to quantify regional myocardial glucose metabolism in normal mice and in those with myocardial infarction (Figure 3). The uptake of FDG reflects the activity of various glucose transporters and hexokinase in manner similar to glucose, but unlike glucose-6-phosphate, FDG6-phosphate is not further metabolized and is trapped into the cells, enhancing imaging quality. This radiotracer is also useful to study inflammation in course of acute myocardial ischemia-reperfusion injury and LV remodeling. As an alternative to glucose metabolism, PET studies with a variety of compounds with similar structure to the fatty acids radiolabeled with $[18 \mathrm{~F}]$ are useful for studying cardiac metabolism, since an ischemic event induces the use of glucose as preferential energy substrate instead of fatty acids, for a long time. C-11 acetate has been proposed as a marker of myocardial viability, because it evaluates residual oxidative metabolism, that is preserved in dysfunctional but viable myocardium, whereas it is severely depressed in irreversibly dysfunctional segments [27]. In the literature it has been described preclinical studies in rat with [18F]-RGD, a cyclic peptide that specifically recognizes receptors $\alpha \nu \beta 3$, and useful to evaluate angiogenesis after myocardial ischemia [36] and with 11C-epinephrine, demonstrating impaired uptake and storage of catecholamines in the myocardium after ischemia induction [37].

\section{Technological Features of Dedicated PET/CT Systems}

The development of PET systems dedicated to small laboratory animals started in the early 1990s. The first dedicated scanner commercialized was the "RatPET" in 1995 [38]. Since then, the main objective has been the improvement of the performances of these devices, considering that heart size is 10 times smaller and heart rate is 10 times faster 
TABLe 2: Principal characteristics of PET tracers for cardiovascular imaging.

\begin{tabular}{|c|c|c|c|c|}
\hline Tracer & Production & Half-life & Extraction & Myocardial uptake mechanism \\
\hline${ }^{13} \mathrm{~N}$-ammonia & Cyclotron & $10 \mathrm{~min}$ & $80 \%$ & Diffusion/metabolic trapping (perfusion) \\
\hline${ }^{82}$ Rubidium & Generator & $78 \mathrm{sec}$ & $50-60 \%$ & $\mathrm{Na} / \mathrm{K}$-ATPase (perfusion) \\
\hline${ }^{15} \mathrm{O}$-water & Cyclotron & $2.1 \mathrm{~min}$ & $100 \%$ & Free diffusion (perfusion) \\
\hline F-18 FDG & Cyclotron & $110 \mathrm{~min}$ & $1-3 \%$ & Glucose transport/hexokinase (viability) \\
\hline
\end{tabular}

PET: positron emission tomography; Na/K-ATPase: sodium/potassium-ATPase; FDG: fluorodeoxyglucose.
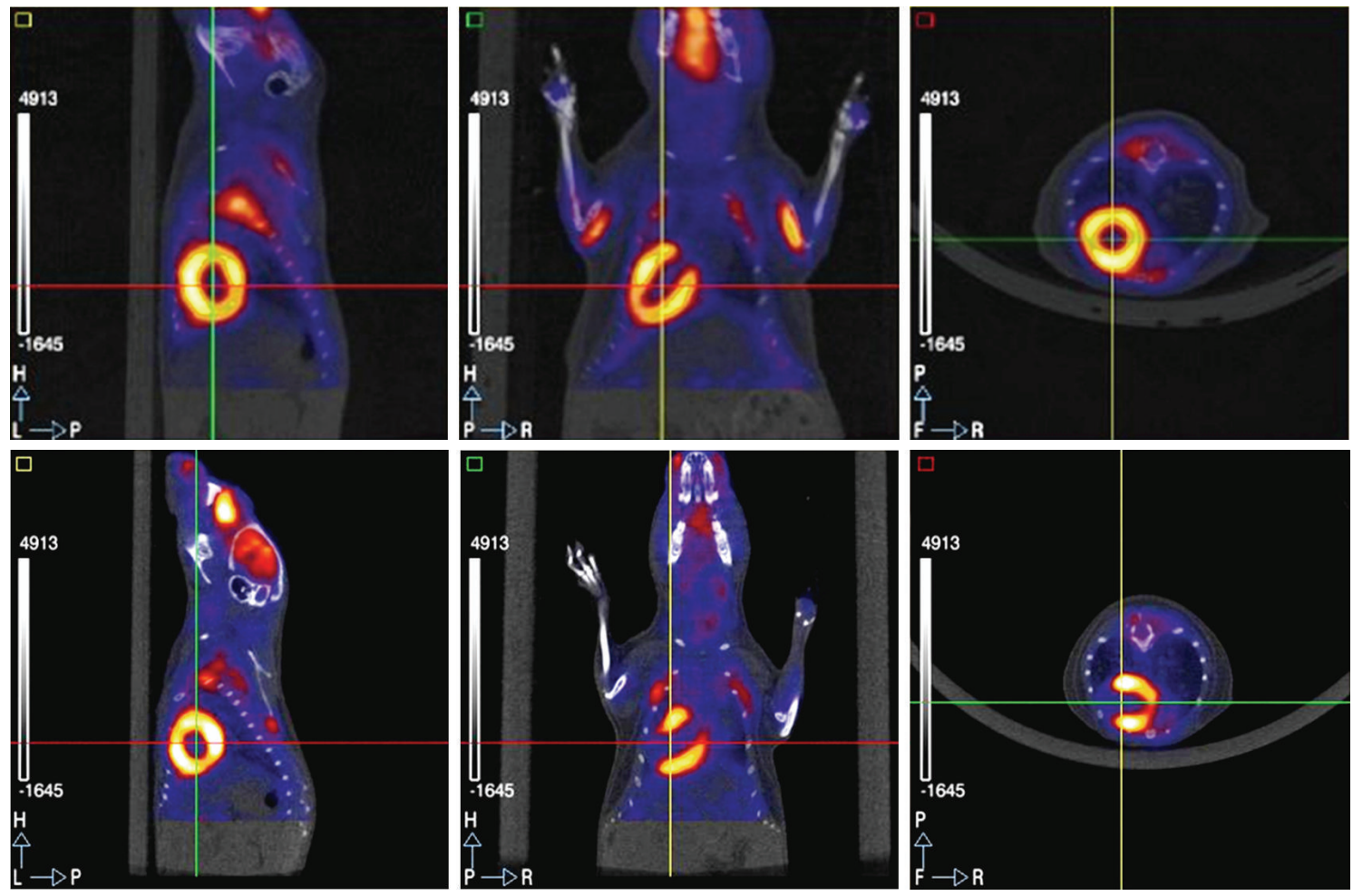

FIGURE 3: $\left[{ }^{18} \mathrm{~F}\right]$ FDG cardiac PET/CT in a normal mouse (top) and in a mouse with myocardial infarction (lower). FDG cardiac uptake is uniform in the normal mouse, while there is an area of absent uptake in the anteroapical region of the mouse with myocardial infarction.

in mice than humans. It is well known that PET is characterized by two intrinsic physical limits to the maximum spatial resolution achievable: the finite range of positron and the acollinearity of the two annihilation photons. It has been estimated that the distance which F-18 travels prior to annihilation produces a blurring effect of about $0.1-0.2 \mathrm{~mm}$, whereas the deviation of annihilation photons from the $180^{\circ}$ direction will produce an additional blur of $0.3 \mathrm{~mm}$ for a $15 \mathrm{~cm}$ detector diameter scanner. Therefore, the highest theoretically resolution achievable with F-18 is about $0.6 \mathrm{~mm}$. Dedicated systems to date available have a spatial resolution between 1 and $2 \mathrm{~mm}$ full width at half maximum (FWHM) [39-41]. Problems related to nonuniformity of spatial resolution can be limited measuring depth of interaction of gamma rays in detectors composed of two joined scintillators with different decay time constant, for example LSO and GSO crystals. Nowadays the small animal scanner with higher spatial resolution is characterized by a detector system formed by layers of laminated lead with several holes that convert the incident gamma rays in electrons, and gas ionization chambers that detect these electrons. This peculiar detector system, associated with the use of a "resolution recovery" reconstruction algorithm, allows to achieve a spatial resolution of $1 \mathrm{~mm}$ FWHM [41]. System sensitivity, defined as the fraction of radioactive decays detected per volumetric resolution elements, is the other important parameter affecting image quality. The detector material, thickness, and geometry are the main factors affecting sensitivity. Currently both human and preclinical PET scanners detect about $2-5 \%$ of the coincident annihilation photons at the center of field of view. The main challenge of dedicated imaging systems is to obtain at the same time a high spatial resolution and sensitivity. The difficulty to reach this goal is in the design and realization of the detectors. To achieve a high spatial resolution in a dedicated PET scanner with inorganic crystals (BGO, $\mathrm{LSO}(\mathrm{Ce}), \mathrm{GSO}(\mathrm{Ce})$ ) detectors, the size of the crystals crosssection must be reduced. As a consequence, the solid angle 
covered by a single detector is limited, decreasing the system sensitivity. Moreover, a reduction of crystals size increases the number of gamma rays obliquely incident on their surface, causing parallax error that degrades spatial resolution from the center toward the edge of the field of view. On the other hand, the increase in the numbers of discrete small crystals poses some difficulties in terms of light collection and readout electronics. Theoretically, the coincidence photon detection efficiency should be improved by a factor of 1000 scaling from clinical to mouse imaging, but it is possible to achieve a 200-fold increase in 2D and a 30-fold increase in 3D data acquisition mode. The resolution-sensitivity offset also strongly affects dynamic scans, because reconstruction of short time frames of PET data may result in unacceptable low signal-to-noise ratio [40]. Iterative statistical algorithms can optimize tradeoff between signal-to-noise ratio and spatial resolution [42]. Image quality is also influenced by the total amount of radioactivity in the field of view, and the scan time. In order to collect an adequate amount of events for good quality image reconstruction, administered dose can be increased securely for probes of endogenous compounds such as the analogue of glucose FDG, overcoming some of the sensitivity limitations. For probes targeting biological markers in nanomolar concentration in tissues, an increase in specific activity allows more radioactivity to be injected into an animal. The number of detected events can be also incremented by long scan time, whereas this approach is limited by radionuclide half-life, radiotracers clearance, and animal anesthesia duration [40]. In molecular imaging, PET provides the possibility to visualize metabolic processes at cellular level noninvasively, quantitatively, and repeatedly, but does not allows a precise anatomic localization of lesions. The introduction of micro-PET/CT hybrid systems for imaging of small animals has greatly enhanced the performance and the accuracy of nuclear imaging, allowing a fine spatial localization of the radiotracer biodistribution and will facilitate the translation of molecular-based imaging to humans. Delayed enhancement micro-CT, using bloodpool and conventional iodinated contrast agent can help to optimize measurement of infarct size, since molecular targets and biological processes differ greatly between necrotic and remote myocardium [29]. Micro-CT can be also useful to perform attenuation correction that can result in underestimation of regional radiotracer activity. Whereas attenuation of the $511 \mathrm{KeV}$ photons is about $22 \%$ for a mouse [41], CT-based attenuation correction has been shown to be accurate in small animal PET, allowing to reduce noise and significantly improve the accuracy of semiquantitative uptake measurements [43]. Several scanner designs are suited for different preclinical research fields. Generally, micro-CT provides higher spatial resolution $(<500 \mu \mathrm{m})$ than current clinical scanner (from 450 to $600 \mu \mathrm{m}$ ). Scaling down CT imaging to the size of a mouse is challenging: to acquire CT data with detail of internal organs comparable to clinical CT scan, a resolution of about $100 \mu \mathrm{m}$ is required. The heart rate of a mouse is about $400-600 \mathrm{~min}^{-1}$ and to use the diastole as the phase of the heart cycle that show the minimum amount of motion, a CT temporal resolution of almost $50 \mathrm{~ms}$ is necessary in comparison to $300 \mathrm{~ms}$ in humans. In order to achieve high spatial and contrast resolution, high X-ray doses are needed, ranging from 250 to $500 \mathrm{mGy}$, in comparison to $<50 \mathrm{mGy}$ for a clinical scanner. Currently, flat-panel-based mini-CT systems offer a valuable tradeoff among resolution $(200 \mu \mathrm{m})$, scan time $(0.5 \mathrm{~s})$, and applied X-ray dose. Although these values remain below the lethal level for the mouse ( $6 \mathrm{~Gy})$, repeated exposure to small X-ray dosages can have biological effects, which might interfere with longitudinal imaging protocols, for example, on tumor growth or hematopoiesis [44].

\section{Animal Preparation for Cardiac PET/CT Imaging}

Parallel to the development of dedicated PET scanners, the need to optimize the operative protocols for small rodents imaging has been profiled. For human F-18 FDG studies, standard protocols have been established, whereas procedures for animal imaging vary widely. In planning longitudinal PET studies in mice, many variables interfering with the accuracy of the experimental results must be taken into account. Several factors may influence the kinetics and biodistribution of radiopharmaceuticals, such as the mouse preparation, the type of radiotracer employed, methods and timing of radiopharmaceutical administration, and anesthesia. Mice attending personnel should be trained for resolutel and gentle manipulation. In fact, stress related to physical restraint can induce corticosteroid and epinephrine release, stimulate cardiovascular and respiratory functions, and increase glucose levels, body temperature, and anesthetic dosages [45]. Moreover, restraint distress during F-18 FDG injection can cause evident uptake of radiotracer in interscapular brown fat and paraspinal muscles, interfering with visualization of heart [45]. Several studies have investigated the effects of fasting, warming, and anesthesia on small animal PET studies with FDG [46]. Under normal resting conditions, myocardium derives the majority of energy from long chain fatty acids, but it can rapidly change their energy source to glucose in response to catecholamine or insulin stimulation, or acute ischemia. It is reported that in fasted mice myocardium should use fatty acids as its major energy source, with consequently reduced glucose or FDG uptake $[47,48]$. In humans, glucose loading and insulin administration are commonly used to improve image quality and diagnostic accuracy. A glucose/insulin stimulation protocol was successfully applied in rats [49] and in mice [50], to standardize metabolic conditions for all animals and produce a 50-fold increase of FDG uptake into the myocardium. This protocol provides intraperitoneal administration of $8 \mathrm{mU} / \mathrm{g}$ of human insulin and $1 \mathrm{mg} / \mathrm{g}$ of glucose thirty minutes before FDG injection, and a biodistribution of thirty minutes prior to a static PET scan of heart. Keeping patients warm from before the FDG injection to the end of PET scanning is a standard practice, commonly adopted for reducing interscapular brown fat uptake [45]. Fueger et al. [46] reported that in mice warming significantly reduces the intense FDG uptake in brown adipose tissue, which could interfere with a clear visualization of heart. In fact, at a so-called "zone 
of thermoneutrality" room temperature $\left(30-34^{\circ} \mathrm{C}\right)$, body temperature is controlled by heat convection and activation of brown adipose tissue and muscle activity are not required to maintain it stable. Anesthesia can influence myocardial metabolism and several haemodynamic parameters, such as myocardial blood flow. Lee et al. [51] compared the effects of ketamine-xylazine and pentobarbital anesthesia on FDG biodistribution in fasted mice. They found that after 4 hours fast, both anesthetic protocols reduced FDG uptake in myocardium. This finding can be explained by the significant increase in plasma glucose level brought by xylazine and by the increased insulin level brought by pentobarbital. Hildebrandt et al. [45] reported that isoflurane, compared to ketamine-xylazine, induces mild hyperglycemia, reduces FDG uptake by brown adipose tissue and increases it in myocardium. Woo et al. [52] observed a dose-dependent increase in FDG uptake in heart under isoflurane anesthesia, likely related to a dose-dependent decrease in blood pressure, proportional to anesthetic depth.

Kober et al. [53] carried out regional myocardial blood flow under two commonly used anesthetic regimens in mice. They reported that under ketamine $100 \mathrm{mg} / \mathrm{kg}$ and xylazine $5 \mathrm{mg} / \mathrm{kg}$ or isoflurane $1.25 \%$ the mean blood flow value in the LV myocardium was comparable. However, under isoflurane concentration of about $2 \%$, myocardial blood flow, and then the uptake of FDG or other radiotracers increased remarkably. In fact, isoflurane has been shown to have effects on blood flow and vascular resistance in many tissues including the heart. Its dose-dependent vasodilatory effect on coronary microvessels has been assessed in vivo in various animal models and in tissue preparations [53] and is based on a mechanism of mitochondrial adenosine triphosphate regulated potassium (mitoK $\mathrm{K}_{\mathrm{ATP}}$ ) channels opening [54]. Ketamine/xylazine could enhance myocardial uptake of radiotracers that interact with catecholamine receptors. Such behavior seems to be due to a reduced blood norepinephrine concentration and radiolabeled analogues activity, brought about by the inhibition of norepinephrine release via $\alpha 2$ adrenoreceptors agonist [55]. The volume of radiotracer bolus should not exceed in mice $200 \mu \mathrm{L}$ for the risk of iatrogenic hypervolemia and lung edema. Therefore, short half-life radionuclides have a maximum time of use in small rodents. Moreover their specific activity decreases over time, increasing the number of molecules injected and the potential appearance of side effects. This problem is critical in mice, because they receive a radiotracer dose greater of humans: for example, in mouse is commonly used a dosage of $0.2 \mathrm{mCi}$ of F-18 FDG, that is, 50 times greater than the human one $(10 \mathrm{mCi})$, taking into account the body weight. This factor is important to improve image quality but is related to a higher radiation dose (about $40 \mathrm{cGy}$ for F-18 PET) [45]. The route of administration is another factor that could influence kinetics and biodistribution of radiopharmaceuticals. In humans, radiotracers are commonly administered intravenously and injection error occurs in about $1 \%$ of patients [56]. The two most common ways used in mice are intravenous and intraperitoneal injection. In preclinical PET studies, intravenous injection is preferred, because the circulation time is faster, the behavior of tracer kinetic is analogue to human and no residual tracer remains in peritoneum. The intraperitoneal route can be associated with modifications in tracer kinetics, wide variability of acquired data, wrong radiotracer injection in internal organs, and interference with abdominal studies. However, due to the very small diameter of murine tail veins, partial paravenous injection is common, leading to a net underestimation of the injected dose. Therefore, like in humans [56], it has been suggested the opportunity to measure residual radioactivity in the tail with a short PET scan to correct the injected dose for radioactivity deposition [57]. Moreover, Fueger et al. [46] found that at 60 minutes after injection, FDG biodistribution is comparable for intravenous and intraperitoneal route.

\section{Postprocessing of PET/CT Images in Models of Myocardial Infarction}

The use of N-13 ammonia, rubidium-82, and F-18 FDG PET for myocardial perfusion and viability imaging in patients with CHD has increased significantly over the last decade [58-60]. PET allows noninvasive measurements of physiological parameters in absolute units or semiquantitative data. The quantitative assessment of regional tracer distribution has represented the main improvement in clinical nuclear cardiology. A reliable, automated, and reproducible analysis of cardiac SPECT or PET data is an important goal and several software programs perform quantitative analysis of myocardial perfusion and viability, according to a scheme divided into some basic steps. First, the myocardial emission activity from the left ventricle is "segmented," that is isolated from any other adjacent emission activity. Since tomographic PET images are usually acquired transaxially to the long axis of the patient, the cardiac images must be reoriented by defining its long axis. Some software programs also allow defining the valve plane and the junction between left and right ventricle. At this point, the tomographic PET data of the heart can be reprocessed into short axis slices (from apex to the base, perpendicularly to the long axis of the heart), vertical long axis slices (from the posterior to anterior wall, parallel to long axis of the heart), horizontal long axis (from the lateral wall to septum, parallel to the long axis of the heart) to help the user in validating the reorientation. Assuming a simplified structural model of the left ventricle, described as a cylindrical structure in its basal two-thirds and spherical at the base, a regular sampling of maximal activity (in 30-60 equally spaced points) is conducted from each short axis section, and along rays normal to the myocardium. These data are used to compute a two-dimensional representation of midmyocardium called "circumferential profile." The epicardial and endocardial surfaces are located at fixed standard deviation values below the midmyocardial maxima. Circumferential profiles are arranged as a series of concentric circles, proportionally to the number of short axis slices, in a "polar map." A polar map is a two-dimensional representation of the threedimensional distribution of the radiotracer in the heart, with the apex corresponding to the center, and the outermost 


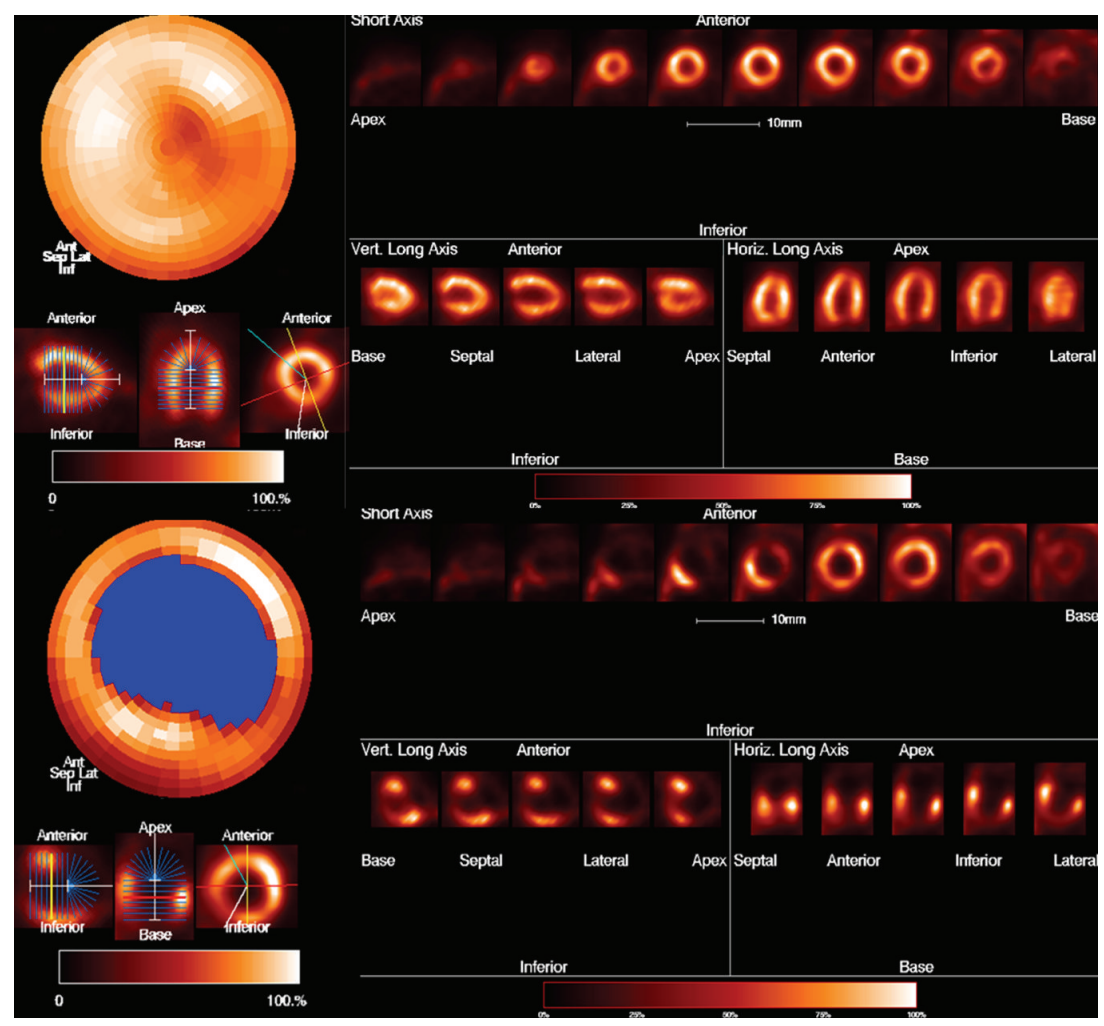

FIGURE 4: $\left[{ }^{18} \mathrm{~F}\right]$ FDG PET. Left ventricular polar map obtained in a normal mouse (top) and in a mouse with myocardial infarction (lower) by MunichHeart software.

circle corresponding to the base of the left ventricle [6163]. Epicardial and endocardial surfaces can be used for displaying $3 \mathrm{D}$ rendering of myocardial perfusion that can be rotated and viewed as a color mapping of perfusion or as a wire-mesh frame. The regional distribution of radiotracer activity in LV myocardium (lateral, anterior, septal, inferior, and apical quadrant of the polar display) can be expressed in $\mathrm{MBq} / \mathrm{mL}$, standard uptake value, and standard deviation from maximal myocardial activity. However, a normal threshold for quantify infarct size has not yet been reported. Several algorithms for quantification of myocardial perfusion are based on the development and validation of a normal database or by measures obtained from anthropomorphic phantoms; others display all perfusion values below 50\% as blackout pixels $[61,63,64]$. Generally polar maps are normalized for maximum activity value and scaled from 0 to maximum activity of $100 \%$. Consequently, defects in perfusion are commonly expressed in percentage value by counting the number of pixels in the polar map whose the activity is below normal threshold and relate this value to the total number of pixels. Moreover, it is possible to determine the standard deviation from the normal threshold value for the pixels whose count is considered below normal [62] or measure infarct size in $\mathrm{cm}^{2}$ using the calculated individual element size $[61,63]$. Blackout pixels in the polar map are used as visual indication of perfusion defect size. Although the quantification of infarct size has been well established in humans with PET, this approach is more challenging in small animals due to their smaller size. In preclinical cardiovascular research, FDG PET is useful to accurately evaluate myocardial glucose metabolism and infarct size, monitoring in vivo and noninvasively the same animal. Its accurate and serial quantification may allow future applications in different knockout and to evaluate postischemic treatments, such as new drugs, gene therapy, or stem cells. The assessment of myocardial perfusion and viability by PET has been described in rodents, with the help of automated software owners [32, 65], clinical software's (Scion Image, ImageJ) $[64,66]$, or dedicated programs $[31,33,49]$. The validation of quantitative PET analysis methods developed for small laboratory rodents has been performed by histomorphometry $[31,49,66]$ or by autoradiography as the gold standard $[32,64]$. The threshold value of normal myocardial perfusion to accurately measure the extent of the necrotic area was determined by linear regression for comparison with histological measurements [46], or based on the method described by O'Connor et al. [67], providing that the pixel values less than $50 \%$ of maximum activity correspond to myocardial hypoperfused areas. Necrotic pattern was defined as relative tracer uptake of $<50 \%$ of maximal myocardial uptake. PET can also be used to measure changes in anatomic and functional parameters, such as LV shape, end-diastolic and end systolic, volumes, stroke volumes, cardiac output, ejection fraction, and regional contractility [32], occurring in postinfarction cardiac remodeling (Figure 4). 


\section{Conclusions}

In the last decades, murine models of MI have become useful tools to highlight the mechanisms underlying CHD and to test new therapeutic approaches, such as gene or stem cells therapy. At the same time, with technological advancements, small laboratory PET/CT imaging has emerged in cardiovascular research, providing in vivo a noninvasive, serial and quantitative assessment of myocardial perfusion and metabolism at a molecular level. PET/CT studies of myocardial perfusion and viability have been successful tools to perform a quantitative evaluation of myocardial metabolism and to measure infarct size in an accurate and repeatable way both in clinical and in preclinical research. Since the same animal can be imaged repeatedly and each animal can be its own control, the number of animals examined is significantly reduced and the variability caused by interindividual differences is removed, according to the principle of "refinement, reduction, and replacement."

\section{Conflict of Interests}

The authors declare no conflict of interests.

\section{References}

[1] F. A. Recchia and V. Lionetti, "Animal models of dilated cardiomyopathy for translational research," Veterinary Research Communications, vol. 31, no. 1, pp. 35-41, 2007.

[2] D. Kumar, T. A. Hacker, J. Buck et al., "Distinct mouse coronary anatomy and myocardial infarction consequent to ligation," Coronary Artery Disease, vol. 16, no. 1, pp. 41-44, 2005.

[3] K. M. Nilles and B. London, "Knockin animal models of inherited arrhythmogenic diseases: what have we learned from them?" Journal of Cardiovascular Electrophysiology, vol. 18, no. 10, pp. 1117-1125, 2007.

[4] B. Ciszek, D. Skubiszewska, and A. Ratajska, "The anatomy of the cardiac veins in mice," Journal of Anatomy, vol. 211, no. 1, pp. 53-63, 2007.

[5] A. Wessels and D. Sedmera, "Developmental anatomy of the heart: a tale of mice and man," Physiological Genomics, vol. 15, pp. 165-176, 2004.

[6] F. L. Meijler, "Atrioventricular conduction versus heart size from mouse to whale," Journal of the American College of Cardiology, vol. 5, no. 2 I, pp. 363-365, 1985.

[7] R. H. Hastings and D. Summers-Torres, "Direct laryngoscopy in mice," Contemporary Topics in Laboratory Animal Science, vol. 38, no. 6, pp. 33-35, 1999.

[8] R. H. Brown, D. M. Walters, R. S. Greenberg, and W. Mitzner, "A method of endotracheal intubation and pulmonary functional assessment for repeated studies in mice," Journal of Applied Physiology, vol. 87, no. 6, pp. 2362-2365, 1999.

[9] A. Vergari, A. Polito, M. Musumeci, S. Palazzesi, and G. Marano, "Video-assisted orotracheal intubation in mice," Laboratory Animals, vol. 37, no. 3, pp. 204-206, 2003.

[10] J. Winning, H. Huwer, J. Redjai et al., "Noninvasive mechanical ventilation of mice by using a balloon-tipped cannula," Contemporary Topics in Laboratory Animal Science, vol. 43, no. 6, pp. 39-41, 2004.
[11] E. N. Spoelstra, C. Ince, A. Koeman et al., "A novel and simple method for endotracheal intubation of mice," Laboratory Animals, vol. 41, no. 1, pp. 128-135, 2007.

[12] O. Moldestad, P. Karlsen, S. Molden, and J. F. Storm, "Tracheotomy improves experiment success rate in mice during urethane anesthesia and stereotaxic surgery," Journal of Neuroscience Methods, vol. 176, no. 2, pp. 57-62, 2009.

[13] O. Tarnavski, J. R. McMullen, M. Schinke, Q. Nie, S. Kong, and S. Izumo, "Mouse cardiac surgery: comprehensive techniques for the generation of mouse models of human diseases and their application for genomic studies," Physiological Genomics, vol. 16, pp. 349-360, 2004.

[14] D. A. Gorog, M. Tanno, A. M. N. Kabir et al., "Varying susceptibility to myocardial infarction among C57BL/6 mice of different genetic background," Journal of Molecular and Cellular Cardiology, vol. 35, no. 6, pp. 705-708, 2003.

[15] J. Takagawa, Y. Zhang, M. L. Wong et al., "Myocardial infarct size measurement in the mouse chronic infarction model: comparison of area- and length-based approaches," Journal of Applied Physiology, vol. 102, no. 6, pp. 2104-2111, 2007.

[16] T. O. Nossuli, V. Lakshminakayanan, G. Baumgarten et al., "A chronic mouse model of myocardial ischemia-reperfusion: essential in cytokine studies," American Journal of Physiology, vol. 278, no. 4, pp. H1049-H1055, 2000.

[17] L. A. Green, S. S. Gambhir, A. Srinivasan et al., "Noninvasive methods for quantitating blood time-activity curves from mouse PET images obtained with fluorine-18-fluorodeoxyglucose," Journal of Nuclear Medicine, vol. 39, no. 4, pp. 729-734, 1998.

[18] H. M. Wu, G. Sui, C. C. Lee et al., "In vivo quantitation of glucose metabolism in mice using small-animal PET and a microfluidic device," Journal of Nuclear Medicine, vol. 48, no. 5, pp. 837-845, 2007.

[19] A. S. Yu, H. D. Lin, S. C. Huang, M. E. Phelps, and H. M. $\mathrm{Wu}$, "Quantification of cerebral glucose metabolic rate in mice using ${ }^{18}$ F-FDG and small-animal PET," Journal of Nuclear Medicine, vol. 50, no. 6, pp. 966-973, 2009.

[20] M. C. Kreissl, H. M. Wu, D. B. Stout et al., "Noninvasive measurement of cardiovascular function in mice with hightemporal-resolution small-animal PET," Journal of Nuclear Medicine, vol. 47, no. 6, pp. 974-980, 2006.

[21] Y. H. D. Fang and R. F. Muzic Jr., "Spillover and partial-volume correction for image-derived input functions for small-animal 18F-FDG PET studies," Journal of Nuclear Medicine, vol. 49, no. 4, pp. 606-614, 2008.

[22] L. W. Locke, S. S. Berr, and B. K. Kundu, "Image-derived input function from cardiac gated maximum a posteriori reconstructed PET images in mice," Molecular Imaging and Biology, vol. 13, no. 2, pp. 342-347, 2011.

[23] J. K. Su, S. L. Jae, C. I. Ki et al., "Kinetic modeling of $3^{\prime}$ deoxy- $3^{\prime}-{ }^{18} \mathrm{~F}$-fluorothymidine for quantitative cell proliferation imaging in subcutaneous tumor models in mice," Journal of Nuclear Medicine, vol. 49, no. 12, pp. 2057-2066, 2008.

[24] L. Moerman, D. de Naeyer, P. Boon, and F. de Vos, "Pglycoprotein at the blood-brain barrier: kinetic modeling of $11 \mathrm{C}$-desmethylloperamide in mice using a 18F-FDG $\mu \mathrm{PET}$ scan to determine the input function," European Journal of Nuclear Medicine and Molecular Imaging, vol. 1, pp. 12-21, 2011.

[25] W. P. Segars, B. M. W. Tsui, E. C. Frey, G. A. Johnson, and S. S. Berr, "Development of a 4-digital mouse phantom for molecular imaging research," Molecular Imaging and Biology, vol. 6, no. 3, pp. 149-159, 2004. 
[26] S. Domenichelli, D. D’Ambrosio, S. Trespidi et al., "Quantitative cardiac dynamic imaging of small animal PET images using cluster analysis," in Proceedings of the Computers in Cardiology (CAR '08), pp. 337-340, Bologna, Italy, September 2008.

[27] J. Kim, P. Herrero, T. Sharp et al., "Minimally invasive method of determining blood input function from PET images in rodents," Journal of Nuclear Medicine, vol. 47, no. 2, pp. 330 336, 2006.

[28] A. Saraste, S. G. Nekolla, and M. Schwaiger, "Cardiovascular molecular imaging: an overview," Cardiovascular Research, vol. 83, no. 4, pp. 643-652, 2009.

[29] M. Nahrendorf, C. Badea, L. W. Hedlund et al., "Highresolution imaging of murine myocardial infarction with delayed-enhancement cine micro-CT," American Journal of Physiology, vol. 292, no. 6, pp. H3172-H3178, 2007.

[30] J. Vogel-Claussen, C. E. Rochitte, K. C. Wu et al., "Delayed enhancement MR imaging: utility in myocardial assessment," Radiographics, vol. 26, no. 3, pp. 795-810, 2006.

[31] T. Wollenweber, C. Zach, C. Rischpler et al., "Myocardial Perfusion Imaging is Feasible for Infarct Size Quantification in Mice Using a Clinical Single-photon Emission Computed Tomography System Equipped with Pinhole Collimators," Molecular Imaging and Biology, vol. 12, no. 4, pp. 427-434, 2009.

[32] L. Stegger, A. N. Hoffmeier, K. P. Schäfers et al., "Accurate noninvasive measurement of infarct size in mice with highresolution PET," Journal of Nuclear Medicine, vol. 47, no. 11, pp. 1837-1844, 2006.

[33] A. Cuocolo, W. Acampa, M. Imbriaco, N. De Luca, G. L. Iovino, and M. Salvatore, "The many ways to myocardial perfusion imaging," Quarterly Journal of Nuclear Medicine and Molecular Imaging, vol. 49, no. 1, pp. 4-18, 2005.

[34] M. Inubushi, M. C. Jordan, K. P. Roos et al., "Nitrogen13 ammonia cardiac positron emission tomography in mice: effects of clonidine-induced changes in cardiac work on myocardial perfusion," European Journal of Nuclear Medicine and Molecular Imaging, vol. 31, no. 1, pp. 110-116, 2004.

[35] M. Ghesani, E. G. DePuey, and A. Rozanski, "Role of F-18 FDG positron emission tomography (PET) in the assessment of myocardial viability," Echocardiography, vol. 22, no. 2, pp. 165-177, 2005.

[36] T. Higuchi, F. M. Bengel, S. Seidl et al., "Assessment of $\alpha \mathrm{v} \beta 3$ integrin expression after myocardial infarction by positron emission tomography," Cardiovascular Research, vol. 78, no. 2, pp. 395-403, 2008.

[37] G. Münch, N. T. B. Nguyen, S. Nekolla et al., "Evaluation of sympathetic nerve terminals with $\left[{ }^{11} \mathrm{C}\right]$ epinephrine and $\left[{ }^{11} \mathrm{C}\right]$ hydroxyephedrine and positron emission tomography," Circulation, vol. 101, no. 5, pp. 516-523, 2000.

[38] P. M. Bloomfield, S. Rajeswaran, T. J. Spinks et al., "The design and physical characteristics of a small animal positron emission tomograph," Physics in Medicine and Biology, vol. 40, no. 6, pp. 1105-1126, 1995.

[39] Y. Wang, J. Seidel, B. M. W. Tsui, J. J. Vaquero, and M. G. Pomper, "Performance evaluation of the GE healthcare eXplore VISTA dual-ring small-animal PET scanner," Journal of Nuclear Medicine, vol. 47, no. 11, pp. 1891-1900, 2006.

[40] M. Larobina, A. Brunetti, and M. Salvatore, "Small animal PET: a review of commercially available imaging systems," Current Medical Imaging Reviews, vol. 2, no. 2, pp. 187-192, 2006.
[41] K. P. Schäfers, A. J. Reader, M. Kriens, C. Knoess, O. Schober, and M. Schäfers, "Performance evaluation of the 32-module quadHIDAC small-animal PET scanner," Journal of Nuclear Medicine, vol. 46, no. 6, pp. 996-1004, 2005.

[42] A. Chatziioannou, J. Qi, A. Moore et al., "Comparison of 3-D maximum a posteriori and filtered backprojection algorithms for high-resolution animal imaging with microPET," IEEE Transactions on Medical Imaging, vol. 19, no. 5, pp. 507-512, 2000.

[43] P. L. Chow, F. R. Rannou, and A. F. Chatziioannou, "Attenuation correction for small animal PET tomographs," Physics in Medicine and Biology, vol. 50, no. 8, pp. 1837-1850, 2005.

[44] J. M. Boone, O. Velazquez, and S. R. Cherry, "Small-animal Xray dose from micro-CT," Molecular Imaging, vol. 3, no. 3, pp. 149-158, 2004.

[45] I. J. Hildebrandt, H. Su, and W. A. Weber, "Anesthesia and other considerations for in vivo imaging of small animals," ILAR Journal, vol. 49, no. 1, pp. 17-26, 2008.

[46] B. J. Fueger, J. Czernin, I. Hildebrandt et al., "Impact of animal handling on the results of $18 \mathrm{~F}-\mathrm{FDG}$ PET studies in mice," Journal of Nuclear Medicine, vol. 47, no. 6, pp. 999-1006, 2006.

[47] M. C. Kreissl, D. B. Stout, K. P. Wong et al., "Influence of dietary state and insulin on myocardial, skeletal muscle and brain [18F]- fluorodeoxyglucose kinetics in mice," European Journal of Nuclear Medicine and Molecular Imaging, vol. 1, no. 6, pp. 1-22, 2011.

[48] T. Higuchi, S. G. Nekolla, A. Jankaukas et al., "Characterization of normal and infarcted rat myocardium using a combination of small-animal PET and clinical MRI," Journal of Nuclear Medicine, vol. 48, no. 2, pp. 288-294, 2007.

[49] G. Münch, N. T. B. Nguyen, S. Nekolla et al., "Evaluation of sympathetic nerve terminals with $\left[{ }^{11} \mathrm{C}\right]$ epinephrine and $\left[{ }^{11} \mathrm{C}\right]$ hydroxyephedrine and positron emission tomography," Circulation, vol. 101, no. 5, pp. 516-523, 2000.

[50] M. V. Simões, S. Egert, S. Ziegler et al., "Delayed response of insulin-stimulated fluorine-18 deoxyglucose uptake in glucose transporter-4-null mice hearts," Journal of the American College of Cardiology, vol. 43, no. 9, pp. 1690-1697, 2004.

[51] K. H. Lee, B. H. Ko, J. Y. Paik et al., "Effects of anesthetic agents and fasting duration on 18F-FDG biodistribution and insulin levels in tumor-bearing mice," Journal of Nuclear Medicine, vol. 46, no. 9, pp. 1531-1536, 2005.

[52] S. K. Woo, T. S. Lee, K. M. Kim et al., "Anesthesia condition for $18 \mathrm{~F}-\mathrm{FDG}$ imaging of lung metastasis tumors using small animal PET," Nuclear Medicine and Biology, vol. 35, no. 1, pp. 143-150, 2008.

[53] F. Kober, I. Iltis, P. J. Cozzone, and M. Bernard, "Myocardial blood flow mapping in mice using high-resolution spin labeling magnetic resonance imaging: influence of ketamine/xylazine and isoflurane anesthesia," Magnetic Resonance in Medicine, vol. 53, no. 3, pp. 601-606, 2005.

[54] H. Toyama, M. Ichise, J. S. Liow et al., "Evaluation of anesthesia effects on [18F]FDG uptake in mouse brain and heart using small animal PET," Nuclear Medicine and Biology, vol. 31, no. 2, pp. 251-256, 2004.

[55] B. H. Ko, J. Y. Paik, K. H. Jung et al., "Effects of anesthetic agents on cellular 123I-MIBG transport and in vivo 123IMIBG biodistribution," European Journal of Nuclear Medicine and Molecular Imaging, vol. 35, no. 3, pp. 554-561, 2008.

[56] K. Miyashita, N. Takahashi, T. Oka et al., "SUV correction for injection errors in FDG-PET examination," Annals of Nuclear Medicine, vol. 21, no. 10, pp. 607-613, 2007.

[57] G. Porenta, W. Kuhle, J. Czernin et al., "Semiquantitative assessment of myocardial blood flow and viability using polar 
map displays of cardiac PET images," Journal of Nuclear Medicine, vol. 33, no. 9, pp. 1628-1636, 1992.

[58] C. Nanni, C. Pettinato, V. Ambrosini et al., "Retro-orbital injection is an effective route for radiopharmaceutical administration in mice during small-animal PET studies," Nuclear Medicine Communications, vol. 28, no. 7, pp. 547-553, 2007.

[59] T. M. Bateman, G. V. Heller, A. I. McGhie et al., "Diagnostic accuracy of rest/stress ECG-gated Rb-82 myocardial perfusion PET: comparison with ECG-gated Tc-99m sestamibi SPECT," Journal of Nuclear Cardiology, vol. 13, no. 1, pp. 24-33, 2006.

[60] U. K. Sampson, S. Dorbala, A. Limaye, R. Kwong, and M. F. Di Carli, "Diagnostic accuracy of rubidium-82 myocardial perfusion imaging with hybrid positron emission tomography/computed tomography in the detection of coronary artery disease," Journal of the American College of Cardiology, vol. 49, no. 10, pp. 1052-1058, 2007.

[61] S. G. Nekolla, C. Miethaner, N. Nguyen, S. I. Ziegler, and M. Schwaiger, "Reproducibility of polar map generation and assessment of defect severity and extent assessment in myocardial perfusion imaging using positron emission tomography," European Journal of Nuclear Medicine, vol. 25, no. 9, pp. 13131321, 1998.

[62] H. Zaidi, Quantitative Analysis in Nuclear Medicine Imaging, Springer, 2006.

[63] G. S. Lin, H. H. Hines, G. Grant, K. Taylor, and C. Ryals, "Automated quantification of myocardial ischemia and wall motion defects by use of cardiac SPECT polar mapping and 4-dimensional surface rendering," Journal of Nuclear Medicine Technology, vol. 34, no. 1, pp. 3-17, 2006.

[64] M. C. Wu, D. W. Gao, R. E. Sievers, R. J. Lee, B. H. Hasegawa, and M. W. Dae, "Pinhole single-photon emission computed tomography for myocardial perfusion imaging of mice," Journal of the American College of Cardiology, vol. 42, no. 3, pp. 576-582, 2003.

[65] T. Kudo, K. Fukuchi, A. J. Annala et al., "Noninvasive measurement of myocardial activity concentrations and perfusion defect sizes in rats with a new small-animal positron emission tomograph," Circulation, vol. 106, no. 1, pp. 118-123, 2002.

[66] P. D. Acton, D. Thomas, and R. Zhou, "Quantitative imaging of myocardial infarct in rats with high resolution pinhole SPECT," International Journal of Cardiovascular Imaging, vol. 22, no. 3-4, pp. 429-434, 2006.

[67] M. K. O’Connor, T. Hammell, and R. J. Gibbons, "In vitro validation of a simple tomographic technique for estimation of percentage myocardium at risk using methoxyisobutyl isonitrile technetium 99m (sestamibi)," European Journal of Nuclear Medicine, vol. 17, no. 1-2, pp. 69-76, 1990. 


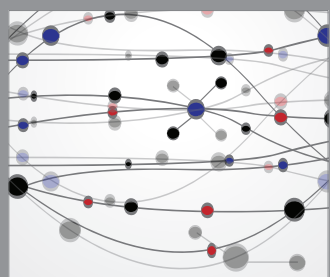

The Scientific World Journal
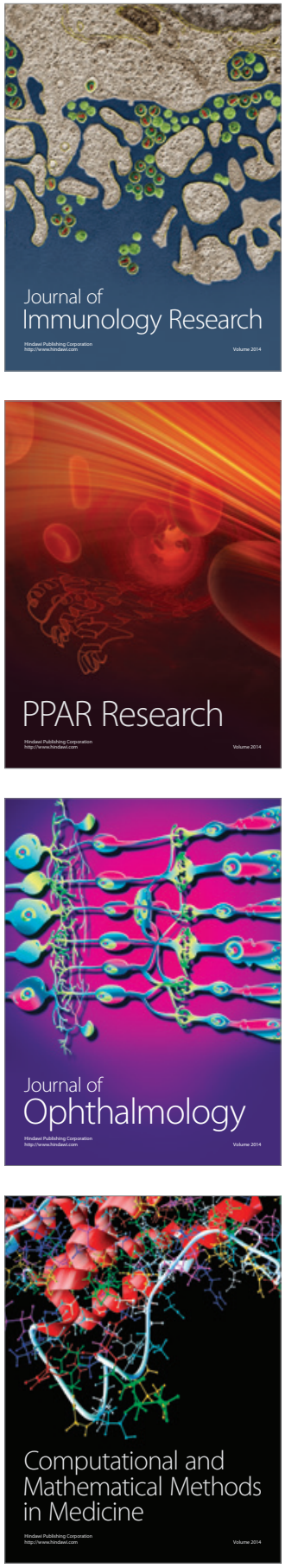

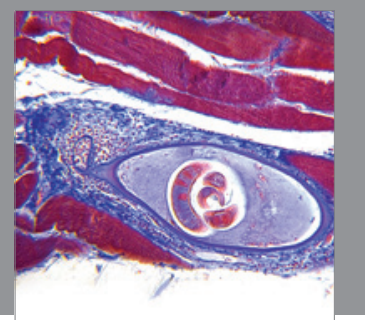

Gastroenterology

Research and Practice
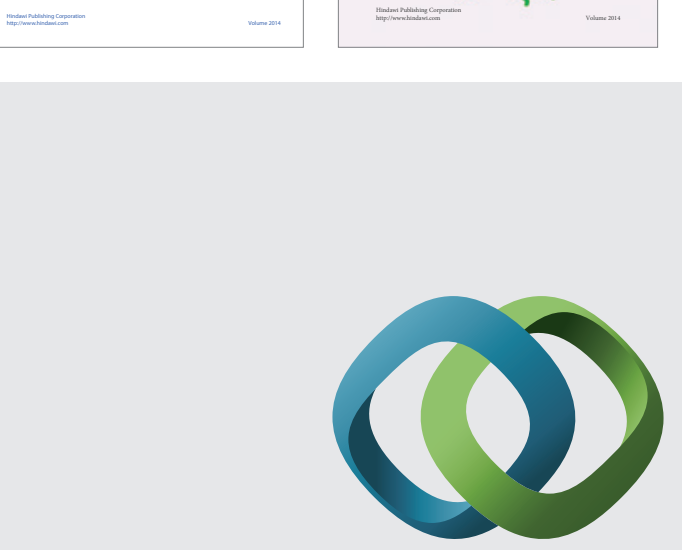

\section{Hindawi}

Submit your manuscripts at

http://www.hindawi.com
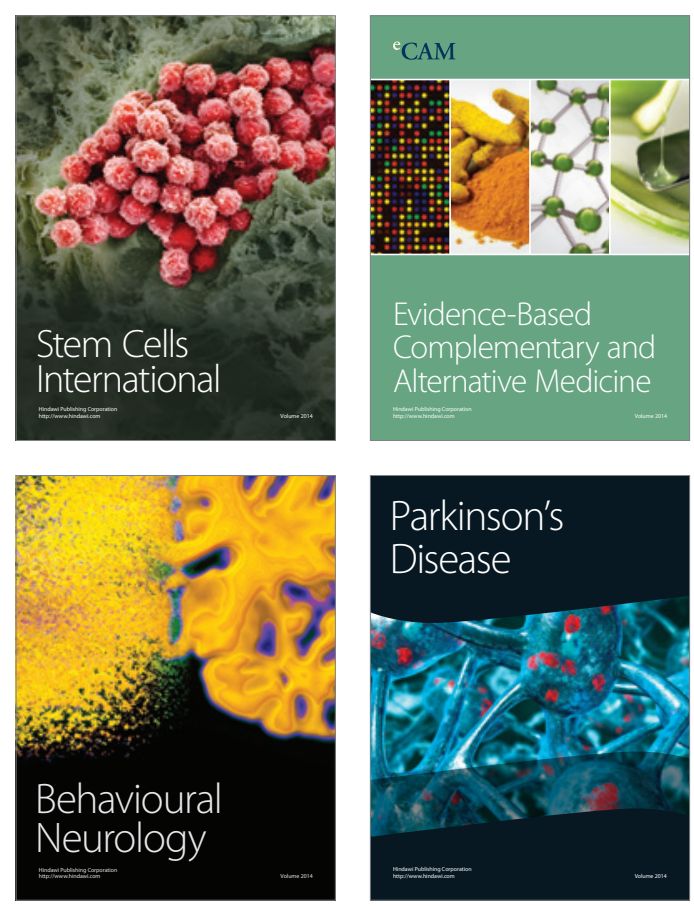

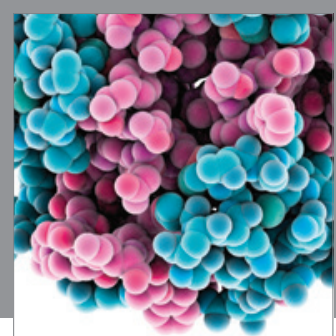

Journal of
Diabetes Research

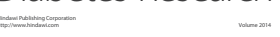

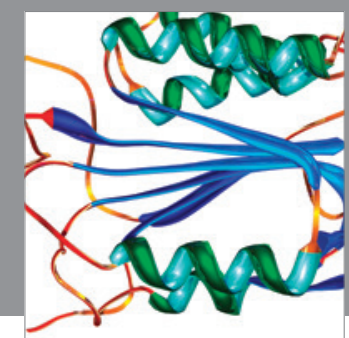

Disease Markers
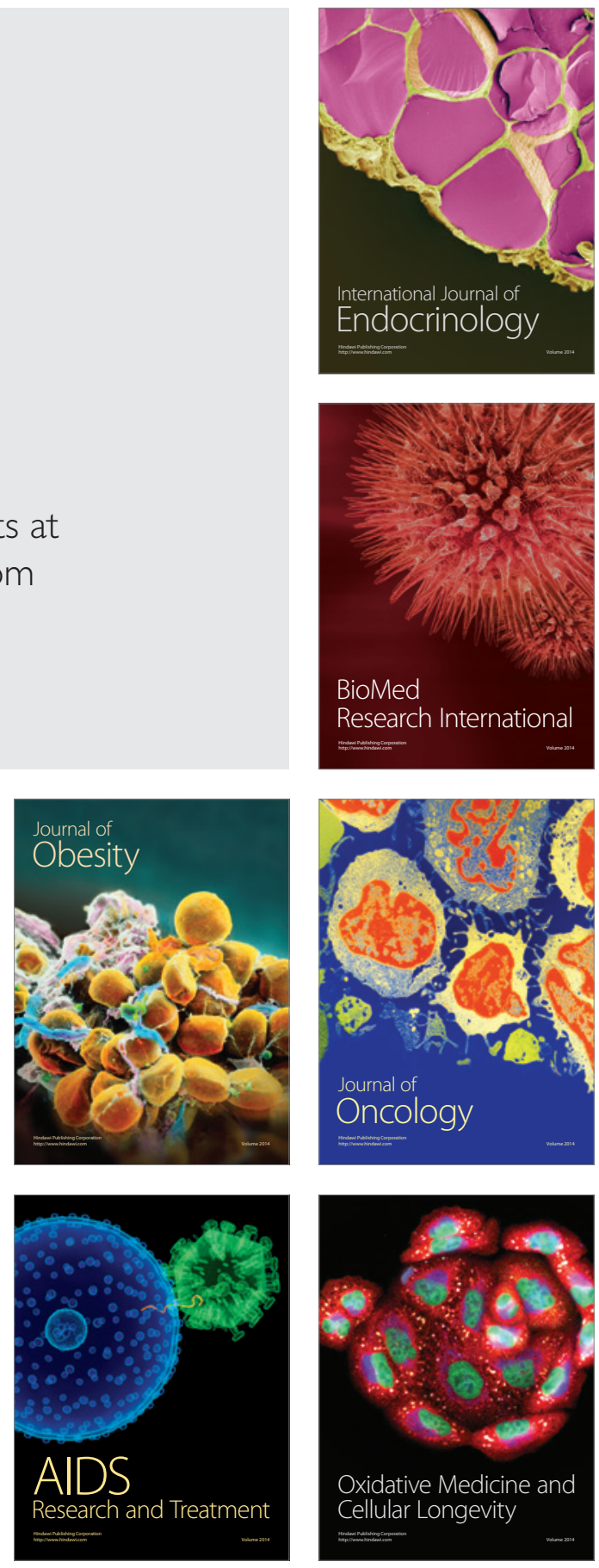